\title{
Designing Analytical Instrument Control Systems for Longevity and Maximum Upward Compatibility
}

\author{
Edward Principe ${ }^{1}$, Anthony D. Owens ${ }^{2}$, William Mershon ${ }^{3}$ \\ 1. Tescan USA, Product Manager FE-SEM and FIB-SEM Products, Warrendale, Pa. USA \\ 2. Tescan USA, Director of Technology, Warrendale, Pa. USA \\ 3. Tescan USA, Applications Manager, Warrendale, Pa. USA
}

Avoiding premature obsolescence of software and hardware which control and communicate with analytical instrument platforms is among the many challenges of instrumentation and facility managers. We will describe design and implementation factors to avoid obsolescence, maximum longevity, upgradeability and reparability.

The most common upgrade of SEMs today is the replacement of controlling PCs running legacy operating systems due to security and supportability issues. An architecture that allows all command and data to pass between the SEM hardware and the controlling PC over a small number of standard interfaces is desirable. The use of Ethernet/TCP/IP for command and data as well as the use of USB for all other interfaces makes it possible to use PCs that are not modified by the inclusion of any special hardware. A robust remote control system that functions over Ethernet makes it easier to guarantee future operation with partners such as EDS analyzers.

To maintain maximum compatibility with current and future Microsoft Windows ${ }^{\mathrm{TM}}$ operating systems, application and instrument control software should make minimal use (if any) of OS-specific database structures such as the Windows registry. Alternative structures for storing information required by instrument control and data acquisition software include the use file types that are more likely to be compatible with current and future operating systems (e.g. ASCII text files or XML files). Instrument control and data acquisition systems should also be designed to minimize or eliminate the need for software drivers (e.g. instrument designs should avoid hardware components that require the use of drivers). Since drivers are typically OS-specific software elements, the use of such hardware components necessitates writing new software drivers to support this hardware when a new OS is employed. In practice, instrument manufacturers often resist investing the time and money to develop new software/drivers that would be required to support older generation tools.

The design of the SEM software user interface can also affect the longevity and ease of upgrading. Most of the changes in software that are required by the need to migrate to newer operating systems come from the behavior of user interfaces. By using the most simple tools available and resisting reliance current fads, it is easier, faster and cheaper to migrate an SEM control application to a newer version of an operating system. The trade-off is not being able to make the interface look new and modern. The benefit is that an interface on Windows ${ }^{\mathrm{TM}} 8$ (or a future Windows ${ }^{\mathrm{TM}} \mathrm{OS}$ ) can have very much the same functionality as the same interface on Windows ${ }^{\mathrm{TM}} 95$ did.

Eluding software and hardware "dead-ends" requires the adoption of standard hardware interfaces and application programmer interfaces. While this can be a difficult task, instrument builders should strive to select and implement communications and data transfer interfaces \& protocols that are likely to be supported in the future as computing platforms evolve. We believe that TCP/IP interfaces will not only 
fulfill that requirement, but also provide additional benefits with respect to system longevity, upgradeability, and reparability:

- TCP/IP will continue as a standard well into the future even as communications/data transfer hardware evolves.

- TCP/IP provides a high speed interface suitable not only for instrument control and query, but for data acquisition and transfer as well, eliminating the need for $3^{\text {rd }}$ party or proprietary image acquisition and/or image data storage ('frame-store') hardware. In practice, such hardware has a limited useful lifetime because if inevitably relies on specific computer bus or hardware interface technologies (e.g. ISA, PCI, Firewire, etc.), as well as commonly requiring OS-specific drivers to function.

- The use of TCP/IP (in essence a pure software interface/protocol) for all instrument control, query, and data acquisition/flow, lends itself to much easier design and implementation of remote clients that can allow user access to instruments over networks as well as remote diagnostic sessions over networks (be these local or wide area networks).

- The combined implementation of TCP/IP and a software API (SEM server process) allows for much more functional, efficient, upgradeable (and bi-directional) interfaces with integrated OEM components (e.g. EDS,WDS, EBSD systems), and minimizes the need for special hardware interfaces that would be more difficult to support in the future.

Finally, we will discuss considerations in electronics design that not only affect instrument longevity, but just as important, affect the time and resource investment (and, therefore, instrument builder's likelihood) to repair or replace electronics that incorporate obsoleted components. Longevity in electronics design is brought about by simplicity. Isolating functions allows the creation of physical modules that have lower parts count, are more compact, and have little or no dependency on the individual performance of other modules. The use of simple low level communications such as $\mathrm{I}_{2} \mathrm{C}$, which have been in wide use for over 30 years, make it easy to consider a module a black box instead of a very specific integrated circuit. This allows a single module to be replaced in the field without the need to adjust any other components. The use of simple, commonly available components maximizes the probability that these components will remain available for use or repair well after they have been declared obsolete by their original manufacturer. It also increases the probability that a newer device can be substituted for the original device. If a module needs to be redesigned, then the scope of work is limited making it less costly for the manufacturer to produce, more likely for customers to purchase, and therefore more likely that both will invest in upgrade of older equipment.

The use of digital signal processors and field programmable gate arrays (DSP/FPGA) allow for the creation of a software-defined SEM. While this constrains a manufacturer to the use of a product line from a single vendor of processors, backward compatibility of software is a primary concern in the design of future DSPs. This makes it more likely that today's development can be recreated in tomorrow's hardware. 